\title{
Cociente ganglionar como factor pronóstico de recurrencia tumoral en pacientes con carcinoma escamoso de cavidad oral
}

\author{
Lymph node ratio as a prognosis factor associated with tumoral recurrence in patients \\ with oral cavity squamous cell carcinoma
}

\begin{abstract}
Absalón Espinoza-Velazco ${ }^{*}$, Adriel S. González-Montoya ${ }^{1}$ y Berenice Carbajal-López ${ }^{2}$
${ }^{1}$ Departamento de Cirugía Oncológica; ${ }^{2}$ Investigación Clínica. Unidad Médica de Alta Especialidad No. 25, Instituto Mexicano del Seguro Social, Monterrey, Nuevo León, México
\end{abstract}

\section{Resumen}

Antecedentes: El cociente ganglionar (LNR) es una herramienta reciente, pero su valor predictivo de recurrencia es incierto. Objetivo: Evaluar el LNR como factor pronóstico de supervivencia libre de enfermedad (SLE) en pacientes con carcinoma oral de células escamosas. Método: Estudio retrospectivo observacional. Pacientes con carcinoma oral de células escamosas sometidos a resección y disección ganglionar. Se aplicaroon las pruebas de ji al cuadrado, Kaplan-Meier, log rank y regresión de Cox; se consideró estadísticamente significativo $p \leq 0.05$ bilateral. Resultados: Fueron incluidos 88 pacientes, el $45 \%(n=40)$ hombres y el $54 \%(n=48)$ mujeres, con una edad media de $60.42( \pm 14.28)$ años. La principal localización tumoral fue la lengua (75\%); el $61 \%$ en etapa I-III y el 39\% en etapa IV. Se dividió en LNR < 0.06 (58\%) y LNR $\geq 0.06$ (42\%). La mediana de SLE no fue alcanzada para ambos grupos $(p=0.018)$. Resultaron predictores de SLE el LNR $(p=0.024$; hazard ratio [HR]: 2.20; intervalo de confianza del 95\% [IC95\%]: 1.11-4.39) y la etapa clínica ( $p=0.004 ;$ HR: 1.76; IC95\%: 1.19-2.59). En el análisis multivariado no se mantuvieron predictores ( $p=0.227$ y $p=0.191$, respectivamente). Conclusiones: Se observaron diferencias significativas en el análisis de SLE, pero no se mantuvieron como predictoras de recurrencia local en el análisis multivariado.

Palabras clave: Carcinoma oral escamoso. Cociente ganglionar.

\begin{abstract}
Background: The lymph node ratio $(L N R)$ is a recent tool, but its predictive value for recurrence is uncertain. Objective: To evaluate LNR as a prognostic factor for disease-free survival (DFS) in patients with oral squamous cell carcinoma. Method: Retrospective observational study. Patients with oral squamous cell carcinoma undergoing resection and lymph node dissection. Chi squared, Kaplan-Meier, log rank and Cox regression tests were run; bilateral $p \leq 0.05$ determined statistical significance. Results: 88 patients were included, $45 \%(n=40)$ men and 54\% $(n=48)$ women, mean age of $60.42( \pm 14.28)$ years. Main tumor location in tongue (75\%); $61 \%$ in clinical stage I-III and 39\% in clinical stage IV. Population was divided into LNR $<0.06$ (58\%) and $L N R \geq 0.06$ (42\%). The median DFS was not reached for both groups $(p=0.018)$. Predictors of DFS were the LNR $(p=0.024$; hazard ratio [HR]: 2.20; confidence interval of 95\% [95\% Cl]: $1.11-4.39)$ and the clinical stage ( $p=0.004 ; \mathrm{HR}: 1.76$; 95\% Cl: 1.19 2.59). In the multivariate analysis, predictors were not maintained ( $p=0.227$ and 0.191 , respectively). Conclusions: Significant differences were observed in the DFS analysis, however, they were not predictive of local recurrence in the multivariate analysis.
\end{abstract}

Key words: Oral squamous carcinoma. Node coefficient.

\section{Correspondencia:}

*Absalón Espinoza-Velazco

Avda. Fidel Velázquez, s/n

Col. Mitras Norte

Fecha de recepción: 26-11-2018

C.P. 64180 , Monterrey, N.L., México

E-mail: dr_absalon@ hotmail.com

0009-7411/@ 2020 Academia Mexicana de Cirugía. Publicado por Permanyer. Este es un artículo open access bajo la licencia CC BY-NC-ND (http://creativecommons.org/licenses/by-nc-nd/4.0/). 


\section{Introducción}

El cáncer oral ocupa la octava posición en el ranking mundial de incidencia de cáncer ${ }^{1,2}$. Cada año, aproximadamente 11 de cada 100,000 adultos son diagnosticados con este tipo de cáncer ${ }^{3-5}$. En México, en 2018, GLOBOCAN reportó una incidencia de 1.48 por cada 100,000 individuos ${ }^{6}$. Actualmente, la octava edición del sistema de clasificación TNM del American Joint Committee on Cancer (AJCC) se utiliza para estadificar y dar pronóstico al carcinoma de células escamosas de cabeza y cuello. La etapificación ganglionar de este sistema se basa en el número, el sitio y el tamaño de los ganglios (nódulos) linfáticos positivos. Pruebas recientes sugieren que, además de estos factores, el número de ganglios positivos y el cociente o la densidad de ganglios linfáticos (LNR o LND, por sus siglas en inglés) son un factor pronóstico crítico que no se incluyó en el sistema de estadificación $\mathrm{TNM}^{7}$. Así, un predictor adicional y emergente para predecir el resultado de los pacientes con cáncer de cavidad oral es la LND o el LNR, que se define como la proporción de ganglios linfáticos positivos con respecto al número total de ganglios linfáticos resecados ${ }^{8}$. En 2009 se realizó un estudio de cohorte con 201 pacientes con diagnóstico de carcinoma de células escamosas aerodigestivo que se sometieron a cirugía y disección de ganglios linfáticos, en el que se determinó el valor pronóstico del LND en la supervivencia global, la supervivencia libre de enfermedad (SLE) y la supervivencia sin falla locorregional. Se halló que la mediana de la supervivencia general fue de 40.70 meses y de 30.11 meses en los pacientes con LNR $\leq 0.06$ y $>0.06$, respectivamente. Además, los autores concluyen que el LNR $>0.06$ es un factor pronóstico significativo para una menor supervivencia general de los pacientes con carcinoma escamoso de cavidad oral. Por tanto, recomiendan tratamientos adyuvantes más intensivos, como quimioterapia con radioterapia y seguimiento a corto plazo para aquellos pacientes con LNR $>0.06^{9}$. Posteriormente, mediante un estudio en 386 pacientes con cáncer de la cavidad oral que se sometieron a tratamiento quirúrgico con o sin terapia adyuvante, se identificó una mediana de LNR de 0.06 como punto de corte relevante y se concluyó que el LNR estaba significativamente asociado con la supervivencia global ( $\leq 0.06$ vs. 0.06 : 58 vs. $28 \%$ ) y con la supervivencia específica de enfermedad ( $\leq 0.06$ vs. $0.06: 65$ vs. $34 \%)$. Además, también se encontró que el LNR era el único predictor significativo del resultado en los pacientes que recibían radioterapia adyuvante. Debido a los altos índices de recurrencia y de mortalidad de la enfermedad en los pacientes con cáncer de cavidad oral, es necesaria la búsqueda de mejores predictores de supervivencia y factores pronósticos de recurrencia ${ }^{10}$.

En este sentido, diversos estudios proponen evaluar el LNR como factor pronóstico independiente para el riesgo de recurrencia local, regional o a distancia del carcinoma oral de células escamosas en pacientes sometidos a disección ganglionar de cuello, y además evaluando otras variables como la etapa patológica, la invasión perineural, la invasión linfovascular, la afección de los márgenes, la extensión extranodal, el subsitio de la cavidad oral, el grosor del tumor, la adyuvancia, el sexo y la edad sobre la recurrencia local, regional o a distancia, para así descartar que los resultados obtenidos sean por estas variables $^{10-14}$.

Por tal motivo se desarrolló el presente estudio, cuyo objetivo fue evaluar el LNR como factor pronóstico independiente asociado a recurrencia en pacientes con carcinoma de cavidad oral de células escamosas sometidos a resección del tumor y disección ganglionar de cuello.

\section{Método}

Estudio de tipo retrospectivo observacional, con muestreo no probabilístico. Se incluyeron pacientes con diagnóstico de carcinoma oral de células escamosas sometidos a resección y disección ganglionar durante el periodo de enero de 2007 a diciembre de 2018 en el servicio de cirugía oncológica del Centro Médico de Noreste, Unidad Médica de Alta Especialidad No. 25 del Instituto Mexicano del Seguro Social; se excluyeron los pacientes con expediente incompleto. Para la realización del estudio se solicitó la autorización al comité de ética e investigación del centro. No fue necesario solicitar el consentimiento informado debido a la naturaleza retrospectiva del estudio. La SLE se definió como el tiempo desde la fecha de la cirugía hasta la última fecha reportada en el expediente clínico.

Para el análisis estadístico se usó el paquete estadístico SPSS V.24, empleando estadística descriptiva e inferencial. Se utilizó la prueba de ji al cuadrado para identificar diferencias entre los grupos. Las pruebas de Kaplan-Meier y log rank fueron empleadas para el análisis de la SLE, y la regresión de Cox para 


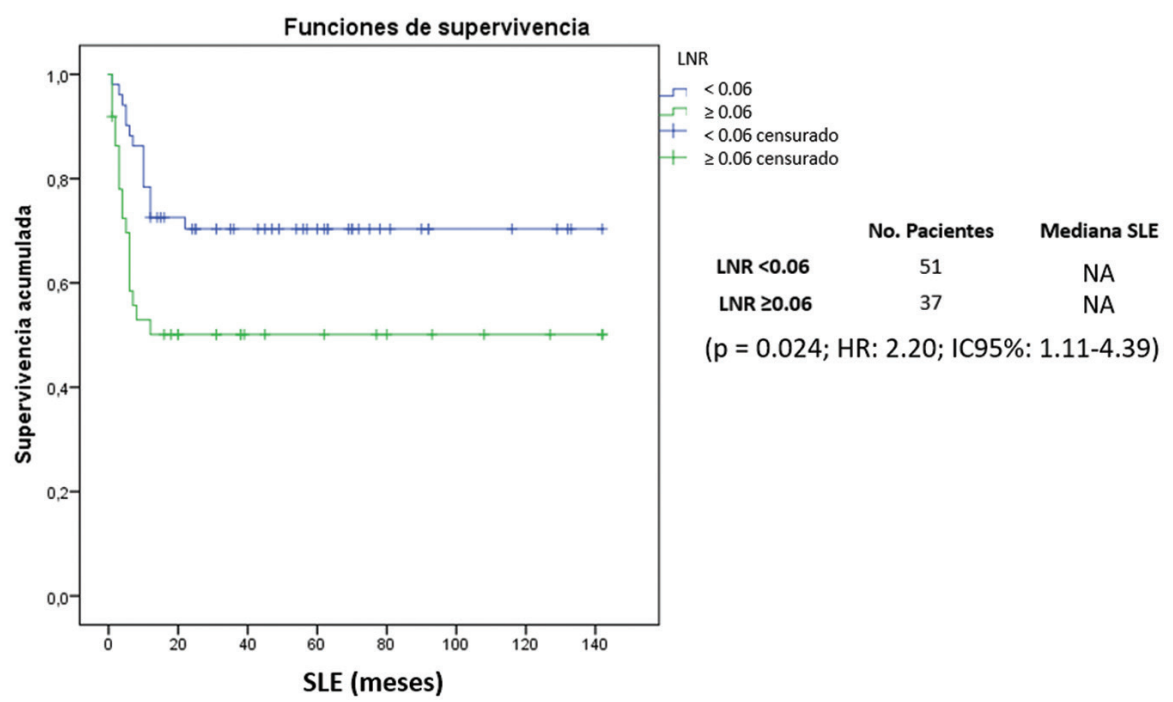

Figura 1. Análisis de la supervivencia libre de enfermedad. HR: hazard ratio; IC95\%: intervalo de confianza del 95\%; LNR: cociente de ganglios linfáticos; NA: no alcanzada; SLE: supervivencia libre de enfermedad.

los análisis univariado y multivariado. Se estableció la significancia estadística con $p \leq 0.05$ bilateral.

\section{Resultados}

Se incluyeron 88 pacientes con diagnóstico de carcinoma oral de células escamosas sometidos a resección tumoral y disección ganglionar de cuello para su análisis. Del total del grupo, el $45 \%(n=40)$ eran hombres y el $54 \%(n=48)$ eran mujeres, con una edad media de $60.42( \pm 14.28)$ años. El principal sitio de localización tumoral fue la lengua (75\%), seguida de las encías (13\%). De acuerdo con la etapificación de la enfermedad, el $61 \%$ de los casos se encontraban en etapas tempranas (I-III) y el restante $39 \%$ se encontraba en etapa clínica IV. En cuanto al estadio de la enfermedad, el T patológico predominante fue T2 (36.8\%, $\mathrm{n}=32)$ y el $\mathrm{N}$ predominante fue NO $(46 \%, \mathrm{n}=40)$.

Los 88 pacientes fueron sometidos a resección quirúrgica y disección ganglionar. La media de ganglios resecados fue de $20.07( \pm 12.55)$, con una mediana de positividad de 1.27 (1-8). El LNR fue $<0.06$ en el $58 \%$ y $\geq 0.06$ en el restante $42 \%$.

Con respecto a los márgenes de disección, el 70\% de los casos presentó R0; adicionalmente, se observó invasión linfovascular y perineural negativa en el $77 \%$ y el $91 \%$ de los casos, respectivamente. En cuanto al grado de diferenciación, el 76\% $(n=67)$ eran de grado 2. Posterior al tratamiento quirúrgico, el $72 \%$ recibió adyuvancia con quimioterapia con 0 sin radioterapia. No se encontraron diferencias entre los grupos al realizar la prueba de ji al cuadrado, exceptuando los ganglios $(N)(p=0.001)$ y la etapa clínica $(p=0.001)$ (Tabla 1).

En el análisis de la SLE, no se alcanzó la mediana de SLE en ninguno de los grupos, con diferencias estadísticamente significativas en la prueba de log rank ( $p=0.018)$ (Fig. 1).

En el análisis univariado se encontraron como predictores de SLE el LNR ( $p=0.024$; hazard ratio [HR]: 2.20; intervalo de confianza del 95\% [IC95\%]: 1.11-4.39) y la etapa clínica ( $p=0.004$; HR: 1.76; IC95\%: 1.19-2.59) (Tabla 2). Por otro lado, en el análisis multivariado, el LNR y la etapa clínica no se mantuvieron como predictores de recurrencia, con $p=0.227$ y $p=0.191$, respectivamente (Tabla 2 ).

\section{Discusión}

El cáncer oral es un problema de salud pública, ya que es una importante causa de morbilidad y mortalidad $^{2}$, y ocupa la octava posición en el ranking mundial de incidencia de cáncer. En México, en 2018, GLOBOCAN reportó una incidencia de 1.48 por cada 100,000 individuos $^{6}$, siendo así el carcinoma oral de células escamosas o cáncer epidermoide el más frecuente de todas las neoplasias orales malignas, abarcando al menos el $90 \%$ de todas ellas ${ }^{2,5,8}$. Posterior al tratamiento inicial, un número importante de pacientes sufre recaídas locales y regionales, por lo que si se lograra identificar un factor pronostico preciso permitirá ofrecer terapias más radicales y hacer un 
Tabla 1. Características generales y resultados clínicos

\begin{tabular}{|c|c|c|c|c|}
\hline \multicolumn{5}{|c|}{ Características clínicas y demográficas } \\
\hline Característica & $\begin{array}{c}\text { LNR }<0.06(n=51) \\
n(\%)\end{array}$ & $\begin{array}{c}\text { LNR } \geq 0.06(n=37) \\
n(\%)\end{array}$ & $\begin{array}{c}\text { Total }(n=88) \\
n(\%)\end{array}$ & $\mathrm{p}$ \\
\hline \multicolumn{5}{|l|}{ Sexo } \\
\hline Hombre & $22(43)$ & $18(49)$ & $40(45)$ & 0.668 \\
\hline Mujer & $29(57)$ & $19(51)$ & $48(54.5)$ & \\
\hline \multicolumn{5}{|l|}{ Edad, años } \\
\hline Media & 59 & 61.38 & 60.42 & 0.440 \\
\hline Desviación estándar & 15.32 & 12.85 & 14.28 & \\
\hline \multicolumn{5}{|l|}{ Localización } \\
\hline Lengua & $38(74)$ & $28(76)$ & $66(75)$ & 0.703 \\
\hline Encías & $6(12)$ & $5(13)$ & $11(12)$ & \\
\hline Piso de cavidad oral & $4(8)$ & $4(11)$ & $8(10)$ & \\
\hline Labio & $3(6)$ & $0(0)$ & $3(3)$ & \\
\hline \multicolumn{5}{|l|}{ Estadio (T) } \\
\hline T1 & $15(27)$ & $8(21)$ & $23(25)$ & 0.156 \\
\hline $\mathrm{T} 2$ & $22(43)$ & $10(27)$ & $32(36)$ & \\
\hline T3 & $9(18)$ & $14(38)$ & $23(25)$ & \\
\hline T4 & $6(12)$ & $5(14)$ & $11(14)$ & \\
\hline \multicolumn{5}{|l|}{ Ganglios (N) } \\
\hline No & $40(78)$ & $0(0)$ & $40(45)$ & $0.001^{*}$ \\
\hline N1 & $9(18)$ & $11(30)$ & $20(23)$ & \\
\hline N2 & $1(2)$ & $25(68)$ & $26(30)$ & \\
\hline N3 & $1(2)$ & $1(3)$ & $2(4)$ & \\
\hline \multicolumn{5}{|l|}{ Etapa clínica } \\
\hline I & $13(26)$ & $0(0)$ & $13(15)$ & $0.001^{*}$ \\
\hline ॥ & $15(29)$ & $0(0)$ & $15(17)$ & \\
\hline III & $15(29)$ & $11(30)$ & $26(29)$ & \\
\hline IV & $8(16)$ & $26(70)$ & $34(39)$ & \\
\hline \multicolumn{5}{|l|}{ Bordes } \\
\hline Ro & $36(71)$ & $26(70)$ & $62(70)$ & 0.961 \\
\hline $\mathrm{R} 1$ & $6(12)$ & $5(13)$ & $11(13)$ & \\
\hline $\mathrm{R} 2$ & $9(17)$ & $6(17)$ & $15(17)$ & \\
\hline \multicolumn{5}{|l|}{ Grado histológico } \\
\hline 1 & $10(20)$ & $4(11)$ & $13(16)$ & 0.535 \\
\hline 2 & $37(72)$ & $30(81)$ & $67(76)$ & \\
\hline 3 & $4(8)$ & $3(8)$ & $7(8)$ & \\
\hline \multicolumn{5}{|l|}{ IV } \\
\hline Negativo & $37(72)$ & $31(84)$ & $68(77)$ & 0.304 \\
\hline Positivo & $14(28)$ & $6(16)$ & $20(23)$ & \\
\hline \multicolumn{5}{|l|}{ IP } \\
\hline Negativo & $48(94)$ & $32(86)$ & $80(91)$ & 0.273 \\
\hline Positivo & $3(6)$ & $5(14)$ & $8(9)$ & \\
\hline Número de ganglios (media) & $20(2-58)$ & $20(2-77)$ & $20.07(2-77)$ & 0.577 \\
\hline Ganglios positivos (mediana) & $1(0-8)$ & $1(0-5)$ & $1(0-8)$ & 0.938 \\
\hline \multicolumn{5}{|l|}{ Tratamiento adyuvante } \\
\hline Sí & $33(65)$ & $30(81)$ & $63(72)$ & 0.102 \\
\hline No & $18(35)$ & $7(19)$ & $25(28)$ & \\
\hline \multicolumn{5}{|l|}{ Recurrencia } \\
\hline Recurrencia & $15(29)$ & $18(49)$ & $33(37)$ & 0.078 \\
\hline Sin enfermedad & $36(71)$ & $19(51)$ & $55(63)$ & \\
\hline
\end{tabular}

LNR: cociente de ganglios linfáticos; IP: Invasión Perineural; IV: Invasión Vascular; P: Valor de significancia estadística; R0: Sin persistencia de enfermedad; R1: Persistencia de enfermedad microscópica; R2: Persistencia de enfermedad macroscópica.

* Significancia estadística $(p<0.05)$. 
Tabla 2. Análisis multivariado. Regresión de Cox

\begin{tabular}{|c|c|c|c|c|}
\hline \multirow[t]{2}{*}{ Variables } & \multicolumn{2}{|c|}{ Univariado } & \multicolumn{2}{|c|}{ Multivariado } \\
\hline & p & HR (IC95\%) & p & HR (IC95\%) \\
\hline$L N R \geq 0.06$ & $0.024^{*}$ & $2.20(1.11-4.39)$ & 0.548 & $1.27(0.57-2.80)$ \\
\hline Localización & 0.750 & $0.95(0.743-1.24)$ & - & - \\
\hline Etapa clínica & $0.004^{*}$ & $1.76(1.19-2.59)$ & 0.95 & $3.84(0.72-18.72)$ \\
\hline Grado 2 & 0.445 & $0.611(0.17-2.16)$ & - & - \\
\hline Bordes & 0.448 & $1.47(0.55-3.75)$ & - & - \\
\hline Invasión vascular & 0.445 & $0.71(0.29-1.72)$ & - & - \\
\hline Invasión perineural & 0.987 & $0.99(0.30-3.24)$ & - & - \\
\hline Adyuvancia & 0.564 & $0.808(0.39-1.66)$ & & \\
\hline
\end{tabular}

HR: hazard ratio; IC95\%: intervalo de confianza del 95\%; LNR: cociente de ganglios linfáticos.

* Significancia estadística $(p<0.05)$.

seguimiento más estrecho de esos pacientes para detectar de forma temprana las recaídas, pudiendo rescatar nuevamente a los pacientes con otras terapias oncológicas y con ello disminuir la morbimortalidad y los gastos en tratamientos y cuidados paliativos que genera la detección tardía de casos. Así pues, un predictor adicional y emergente del resultado de los pacientes con cáncer de cavidad oral es el LNR. Varios estudios retrospectivos han informado que el LNR es predictivo para la supervivencia global ${ }^{12,13-20}$, la supervivencia específica de la enfermedad ${ }^{13-18}$, la SLE $^{13,18,21}$ y el control locorregional ${ }^{16,19,21}$. En el presente estudio se observaron diferencias significativas en el análisis de la SLE ( $p=0.018)$ entre los pacientes con LNR $<0.06$ y aquellos con LNR $\geq 0.06$.

Gil, et al. ${ }^{9}$ evaluaron el papel del LNR en el carcinoma de cavidad oral mediante un estudio en 386 pacientes con cáncer de la cavidad oral. En el análisis multivariado, el LNR (punto de corte de 0.06) mostró ser el único predictor independiente para falla locorregional. En el estudio de Prabhu, et al. ${ }^{19}$ se incluyeron 350 pacientes y se halló que el LNR se asoció significativamente con la recurrencia locorregional al emplear un punto de corte de 0.2; así, los pacientes con una LNR mayor o igual que este valor tenían un alto riesgo de recurrencia locorregional. Igualmente, Lieng, et al. ${ }^{22-24}$ determinaron la importancia pronóstica del LNR en 72 pacientes con carcinoma oral de células escamosas y ganglios positivos. Encontraron que los pacientes con un LNR > 0.143 experimentaron una mayor tasa de recurrencia regional.

En el presente estudio se encontraron como predictores de SLE el LNR y la etapa clínica. Sin embargo, en el análisis multivariado, el LNR y la etapa clínica no se mantuvieron como predictores de recurrencia.

Nuestro estudio presenta algunas limitaciones, como su naturaleza retrospectiva y el tamaño de la muestra. Por ello, se sugiere ampliar el tamaño muestral y hacer un estudio que incluya el análisis por etapa de la enfermedad en relación con el tipo de resección y el LNR.

\section{Conclusiones}

El presente estudio es de gran utilidad ya que, aun cuando el LNR no se mantuvo como predictor único de SLE, podría ser una herramienta que ayude en la determinación del riesgo de recurrencia locorregional en pacientes con carcinoma de cavidad oral. Por tal motivo, sería necesario ampliar la muestra para mejorar la estimación y replicar el análisis incluyendo un subanálisis por etapa clínica de la enfermedad.

\section{Conflicto de intereses}

Los autores declaran que no tienen conflicto de intereses alguno con esta publicación.

\section{Financiamiento}

La presente investigación no obtuvo beneficio económico.

\section{Responsabilidades éticas}

Protección de personas y animales. Los autores declaran que para esta investigación no se han 
realizado experimentos en seres humanos ni en animales.

Confidencialidad de los datos. Los autores declaran que han seguido los protocolos de su centro de trabajo sobre la publicación de datos de pacientes.

Derecho a la privacidad y consentimiento informado. Los autores declaran que en este artículo no aparecen datos de pacientes.

\section{Bibliografía}

1. Neville BW, Day TA. Oral cancer and precancerous lesions. CA Cancer $\mathrm{J}$ Clin. 2002;52:195-215.

2. Saito M, Kobyashi T, Takagi R, Saku T. Clinicopathological distinction of two categories of oral squamous cell carcinoma of the tongue: de novo vs. sequential types. Oral Med Pathol. 2012;16:81-8.

3. Joseph LJ, Goodman M, Higgins K, Pilai R, Ramalingam SS, Magliocca K, et al. Racial disparities in squamous cell carcinoma of the oral tongue among women: a SEER data analysis. Oral Oncol. 2015;51:586-92.

4. Warnakulasuriya S. Global epidemiology of oral and oropharyngeal cancer. Oral Oncol. 2009;45:309-16.

5. Edwards BK, Edwards BK, Noone AM, Mariotto AB, Simard EP, Boscoe FP, Henley SJ, et al. Annual Report to the Nation on the status of cancer, 1975-2010, featuring prevalence of comorbidity and impact on survival among persons with lung, colorectal, breast, or prostate cancer: 2013 Report on the Status of Cancer. Cancer. 2014; 120:1290-314

6. Interational Agency for Research on Cancer (IARC), a dependency of World Health Organization. GLOBOCAN 2018 cancer today. Cancer incidence and mortality worldwide. Lyon, France. Disponible en: https:// gco.iarc.fr/today/online

7. Cramer JD, Reddy A, Ferris RL, Duvvuri U, Samant S. Comparison of the seventh and eighth edition American Joint Committee on cancer oral cavity staging systems. Laryngoscope. 2018;128:2351-60.

8. Eshraghi Samani R, Shirkhoda M, Hadji M, Beheshtifard F, Hamedani SMMG, Momen A, et al. The prognostic value of lymph node ratio in survival of head-and-neck squamous cell carcinoma. J Res Med Sci. 2018;23:35.

9. Gil Z, Carlson DL, Boyle JO, Kraus DH, Shah JP, Shaha AR, et al. Lymph node density is a significant predictor of outcome in patients with oral cancer. Cancer. 2009;115:5700-10.
10. Kim SY, Nam SY, Choi SH, Cho KJ, Roh JL. Prognostic value of lymph node density in node-positive patients with oral squamous cell carcinoma. Ann Surg Oncol. 2011;18:2310-7.

11. Feng Z, Xu QS, Wang C, Li JZ, Mao MH, Li H, et al. Lymph node ratio is associated with adverse clinicopathological features and is a crucial nodal parameter for oral and oropharyngeal cancer. Sci Rep. 2017;7:6708.

12. Liao CT, Hsueh C, Lee LY, Lin CY, Fan KH, Wang HM, et al. Neck dissection field and lymph node density predict prognosis in patients with oral cavity cancer and pathological node metastases treated with adjuvant therapy. Oral Oncol. 2012;48:329-36.

13. Costa Ade L, Araújo Júnior RF, Ramos CC. Correlation between TNM classification and malignancy histological feature of oral squamous cell carcinoma. Braz J Otorhinolaryngol. 2005;71:181-7.

14. Massano J, Regateiro FS, Januário G, Ferreira A. Oral squamous cell carcinoma: review of prognostic and predictive factors. Oral Surg Oral Med Oral Pathol Oral Radiol Endod. 2006;102:67-76.

15. Patel SG, Amit M, Yen TC, Liao CT, Chaturvedi P, Agarwal JP, et al. Lymph node density in oral cavity cancer: results of the International Consortium for Outcomes Research. Br J Cancer. 2013;109:2087-95.

16. Shrime MG, Ma C, Gullane PJ, Gilbert RW, Irish JC, Brown DH, et al. Impact of nodal ratio on survival in squamous cell carcinoma of the oral cavity. Head Neck. 2009;31:1129-36.

17. Kim SY, Nam SY, Choi SH, Cho KJ, Roh JL. Prognostic value of lymph node density in node-positive patients with oral squamous cell carcinoma. Ann Surg Oncol. 2011;18:2310-7.

18. Lanzer M, Kruse A, Lübbers HT, Zemann W, Reinisch S. Lymph node ratio and capsule penetration as independent risk factors in head and neck squamous cell carcinoma. Head \& neck oncology, 2012;4(5):89.

19. Prabhu RS, Hanasoge S, Magliocca KR, Hall WA, Chen SA, Higgins KA, et al. Lymph node ratio influence on risk of head and neck cancer locoregional recurrence after initial surgical resection: implications for adjuvant therapy. Head Neck. 2015;37:777-82.

20. Rudra S, Spiotto MT, Witt ME, Blair EA, Stenson K, Haraf DJ. Lymph node density - prognostic value in head and neck cancer. Head Neck. 2014;36:266-72.

21. Amar A, Rapoport A, Curioni OA, Dedivitis RA, Cernea CR, Brandão LG. A densidade do linfonodo metastático como fator prognóstico no carcinoma espinocelular da língua e soalho bucal. Braz J Otorhinolaryngol. 2012;78:86-90.

22. Lieng H, Gebski VJ, Morgan GJ, Veness MJ. Important prognostic significance of lymph node density in patients with node positive oral tongue cancer: lymph node density in tongue cancer. ANZ J Surg. 2016;86:681-6.

23. Reinisch S, Kruse A, Bredell M, Lübbers HT, Gander T, Lanzer M. Is lymph-node ratio a superior predictor than lymph node status for recurrence-free and overall survival in patients with head and neck squamous cell carcinoma? Ann Surg Oncol. 2014;21:1912-8.

24. Muñoz Guerra MF, Naval Gías L, Campo FR, Pérez JS. Marginal and segmental mandibulectomy in patients with oral cancer: a statistical analysis of 106 cases. J Oral Maxillofac Surg. 2003;61:1289-96. 